\title{
Global Formation of Topological Defects in the Multiferroic Hexagonal Manganites
}

\author{
Q. N. Meier, ${ }^{1}$ M. Lilienblum, ${ }^{1}$ S. M. Griffin, ${ }^{2}$ K. Conder, ${ }^{3}$ E. Pomjakushina, ${ }^{3}$ Z. Yan, ${ }^{4,5}$ E. Bourret, ${ }^{4}$ \\ D. Meier, ${ }^{6}$ F. Lichtenberg, ${ }^{1}$ E. K. H. Salje, ${ }^{7}$ N. A. Spaldin, ${ }^{1}$ M. Fiebig, ${ }^{1}$ and A. Cano ${ }^{1,8}$ \\ ${ }^{1}$ Department of Materials, ETH Zurich, 8093 Zürich, Switzerland \\ ${ }^{2}$ Department of Physics, University of California Berkeley, Berkeley, California 94720, USA \\ and Molecular Foundry, Lawrence Berkeley National Laboratory, Berkeley, California 94720, USA \\ ${ }^{3}$ Laboratory for Scientific Developments and Novel Materials, \\ Paul Scherrer Institute, 5232 Villigen, Switzerland \\ ${ }^{4}$ Materials Sciences Division, Lawrence Berkeley National Laboratory, Berkeley, California 94720, USA \\ ${ }^{5}$ Department of Physics, ETH Zurich, Otto-Stern-Weg 1, 8093 Zürich, Switzerland \\ ${ }^{6}$ Department of Materials Science and Engineering, \\ Norwegian University of Science and Technology, 7491 Trondheim, Norway \\ ${ }^{7}$ Department of Earth Sciences, University of Cambridge, Cambridge CB2 3EQ, United Kingdom \\ ${ }^{8}$ CNRS, University of Bordeaux, ICMCB, UPR 9048, 33600 Pessac, France
}

(Received 13 April 2017; published 20 October 2017)

\begin{abstract}
The spontaneous transformations associated with symmetry-breaking phase transitions generate domain structures and defects that may be topological in nature. The formation of these defects can be described according to the Kibble-Zurek mechanism, which provides a generic relation that applies from cosmological to interatomic length scales. Its verification is challenging, however, in particular at the cosmological scale where experiments are impractical. While it has been demonstrated for selected condensed-matter systems, major questions remain regarding, e.g., its degree of universality. Here, we develop a global Kibble-Zurek picture from the condensed-matter level. We show theoretically that a transition between two fluctuation regimes (Ginzburg and mean field) can lead to an intermediate region with reversed scaling, and we verify experimentally this behavior for the structural transition in the series of multiferroic hexagonal manganites. Trends across the series allow us to identify additional intrinsic features of the defect formation beyond the original Kibble-Zurek paradigm.
\end{abstract}

DOI: 10.1103/PhysRevX.7.041014

\section{INTRODUCTION}

Topological defects are ubiquitous in nature, emerging in various forms in a large variety of physical systems from atomic to cosmic length scales. In the context of cosmology, Kibble first inspected the link between the possible topology of the corresponding defects and gauge symmetry breaking [1]. Subsequently, Zurek derived a scaling law relating the density of defects and the speed at which the transition point is crossed [2]. Their combined theory is known as the Kibble-Zurek mechanism. Under the appropriate conditions, this mechanism is expected to describe the formation of topological defects in a system that is driven through a continuous phase transition at a finite cooling rate. Since Kibble-Zurek scaling is determined by the critical behavior and should be the same for all systems

Published by the American Physical Society under the terms of the Creative Commons Attribution 4.0 International license. Further distribution of this work must maintain attribution to the author(s) and the published article's title, journal citation, and DOI.
Subject Areas: Condensed Matter Physics, Interdisciplinary Physics, Materials Science in the same universality class, Zurek proposed the study of condensed-matter analogues to cosmic systems for its verification.

A variety of condensed-matter systems have been investigated to date in an effort to verify the Kibble-Zurek mechanism. Early attempts were carried out on liquid crystals [3,4], superfluid ${ }^{4} \mathrm{He}$ and ${ }^{3} \mathrm{He}$ [5-8], and superconducting rings $[9,10]$. More recent studies have been conducted on multiferroics [11-13], Bose-Einstein condensates [14,15], ionic crystals [16,17], Landau-Zener setups [18], and colloidal monolayers [19]; for a review, see Ref. [20]. The case of multiferroics is particularly interesting, as they have provided the first experimental setting clearly compatible with a Kibble-Zurek scaling beyond mean field [11]. On the other hand, for the same system, a drastic reversal of this scaling (termed "antiKibble-Zurek scaling" [11]) has been reported for fast quenches, although its origin is not understood and its existence has been questioned [13].

In this work, we combine first-principles calculations and the theory of critical phenomena to provide a global picture of the Kibble-Zurek mechanism in which, by 
increasing the cooling rate, defect formation evolves from the fluctuation-dominated Ginzburg region to the mean-field regime, with the Ginzburg temperature as the energy scale for this crossover. This picture naturally encompasses features of anti-Kibble-Zurek behavior, which can emerge from the crossover between these two distinct regimes.

The possibility that a system can evolve from mean field to a borderline regime beyond mean-field behavior was already recognized in connection to inhomogeneous coolings [21]. Even earlier, the role of the Ginzburg temperature in providing a special energy scale was grasped by Kibble, even though he rather related it to the energy scale determining the formation of topological defects $[1,22]$. [The defect formation is actually determined by the (critical) dynamics.] Finally, continuation of defect formation after reaching the Ginzburg regime has also been demonstrated $[11,13,23]$.

Despite all these spotlights on the difference of fluctuation regimes in relation to Kibble-Zurek physics, an overarching model interrelating these fluctuation regimes and describing the transfer in between them within the Kibble-Zurek formalism has not been developed. Here, we accomplish this, employing the series of hexagonal multiferroic manganites, $R \mathrm{MnO}_{3}$, here with $R=\mathrm{Y}, \mathrm{Dy}, \mathrm{Er}$, and $\mathrm{Tm}$, as a model system for this investigation.

In our scanning probe measurements, both the KibbleZurek scaling and the anti-Kibble-Zurek behavior are demonstrated unequivocally as a general feature in hexagonal manganites. In addition, trends that we uncover by studying the $R \mathrm{MnO}_{3}$ series as a whole reveal additional quantitative features suggesting that the topological defect formation is affected by supplementary ingredients beyond the original Kibble-Zurek theory. We discuss the emergence of additional time scales and length scales as likely candidates for these extra features, which can appear naturally from the propagation of the phase-transition front, the vortex-growth process, or directly from the eventual discrete nature of the corresponding symmetry breaking.

\section{RESULTS}

\section{A. Preliminaries}

We prepare single crystals of $\mathrm{YMnO}_{3}, \mathrm{DyMnO}_{3}$, and $\mathrm{ErMnO}_{3}$ using the floating-zone (FZ) technique as described in Appendixes A and B. To complete our analysis, we also consider data for $\mathrm{TmMnO}_{3}$ reported in Ref. [13]. These $R \mathrm{MnO}_{3}$ compounds undergo a hightemperature lattice-distortive unit-cell-trimerizing transition at $T_{c}^{\mathrm{Y}} \simeq 1259 \mathrm{~K}, T_{c}^{\mathrm{Dy}} \simeq 1223 \mathrm{~K}, T_{c}^{\mathrm{Er}} \simeq 1429 \mathrm{~K}$, and $T_{c}^{\mathrm{Tm}} \simeq 1514 \mathrm{~K}[12,24]$. By driving the systems through this structural transition, topological defects are created in the peculiar form of discrete vortices, as sketched in Fig. 1 [11-13,24-27]. These vortices correspond to particular solutions of the Landau free energy [27],
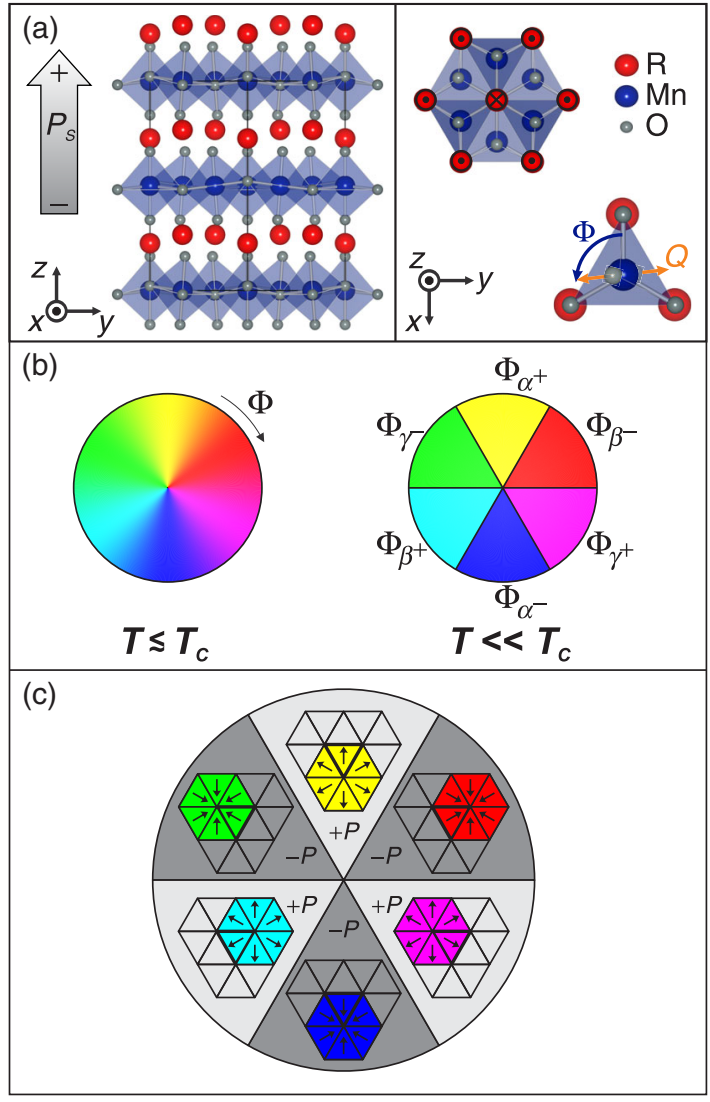

FIG. 1. Formation of topological defects in the hexagonal manganites. (a) Side and top views of the unit cell showing the arrangement of the tilted $\mathrm{MnO}_{5}$ bipyramids. At the ordering temperature $T_{c}$, sets of three bipyramids tilt towards or (in this case) away from a common center. This trimerizes the unit cell and induces a spontaneous polarization $\pm P$. The order parameter of the trimerization polarization is $(Q \cos \Phi, Q \sin \Phi)$ with $Q$ and $\Phi$ as sketched. (b) Topological defects are lines (points on the sample surface) around which $\Phi$ changes monotonically in a clockwise or counterclockwise fashion. Close to $T_{c}$, this change is gradual due to the "dangerously irrelevant" character of the $Z_{6}$ anisotropy. At lower temperature, the $Z_{6}$ anisotropy becomes fully relevant and six domain states with discrete values $\Phi=n \times 60^{\circ}, n=0,1, \ldots, 5$, emerge. (c) Possible arrangement of the six domain states around the vortex-like topological defect. For each domain state the bipyramidal tilt pattern is indicated with arrows representing $Q$ and $\Phi$.

$$
\begin{aligned}
F= & \frac{a}{2} Q^{2}+\frac{b}{4} Q^{4}+\frac{1}{6}\left(c+c^{\prime} \cos 6 \Phi\right) Q^{6} \\
& +\frac{s}{2}\left[(\nabla Q)^{2}+Q^{2}(\nabla \Phi)^{2}\right],
\end{aligned}
$$

where $\mathbf{Q}=(Q \cos \Phi, Q \sin \Phi)$ is the primary order parameter associated with the condensation of a zone-boundary $K_{3}$ phonon. This condensation induces the spontaneous polarization $P \sim Q^{3} \cos 3 \Phi \quad(\Phi=n \pi / 3$, with $n=0$, $1, \ldots, 5)$. The polarization alternation of the resulting six trimerization-polarization domain states around the vortices 
TABLE I. Parameters of the Landau free energy Eq. (1) obtained from DFT calculations (see Appendix C). Here, $a_{0}$ denotes the zero-temperature value of the parameter $a$ [in the simplest case, $a=-a_{0} T_{c} \varepsilon$, where $\varepsilon=\left(T-T_{c}\right) / T_{c}$ is the reduced temperature]. The parameter $s$ in Eq. (1) corresponds to the averaged stiffness $s=\left(s_{x}^{2} s_{z}\right)^{1 / 3}$. The zero-temperature correlation length $\xi_{0}$, renormalized zero-temperature correlation length $\tilde{\xi}_{0}$, and Ginzburg number Gi are derived from these values.

\begin{tabular}{lcccc}
\hline \hline & $\mathrm{YMnO}_{3}$ & $\mathrm{DyMnO}_{3}$ & $\mathrm{ErMnO}_{3}$ & $\mathrm{TmMnO}_{3}$ \\
\hline$a_{0}\left(\mathrm{eV} / \mathrm{A}^{2}\right)$ & -3.2 & -3.6 & -3.8 & -3.9 \\
$b\left(\mathrm{eV} / \mathrm{A}^{2}\right)$ & 5.6 & 5.8 & 6.1 & 5.6 \\
$s_{x}=s_{y}(\mathrm{eV})$ & 4.57 & 4.47 & 4.73 & 4.52 \\
$s_{z}(\mathrm{eV})$ & 17.2 & 18.7 & 18.9 & 20.18 \\
$\xi_{0}(\AA)$ & 1.48 & 1.50 & 1.41 & 1.40 \\
$\tilde{\xi}_{0}(\AA)$ & 2.00 & 2.00 & 1.90 & 1.89 \\
$\mathrm{Gi}(\bar{s}=s)$ & 0.27 & 0.24 & 0.28 & 0.27 \\
$\mathrm{Gi}\left(\bar{s}=s_{z}\right)$ & 0.017 & 0.015 & 0.018 & 0.014 \\
\hline \hline
\end{tabular}

[see Fig. 1(c)] enables their real space imaging by piezoresponse force microscopy $(\mathrm{PFM})$. The parameters of Eq. (1) for the series of hexagonal manganites calculated in this work using density functional theory (DFT) (see Appendix C) are given in Table I.

\section{B. Quenching experiments}

As detailed in Appendix B, suitable sets of samples are prepared by preannealing a larger set of samples above the respective transition temperature and selecting only those samples that reveal, for every compound separately and within statistical error, the same density of vortices under identical annealing conditions. In addition, we verify in $\mathrm{YMnO}_{3}$ samples that they lose any memory of the ferroelectric preannealing domain state after exceeding $T_{c}$ by at least $20 \mathrm{~K}$. For a cooling rate of $5 \mathrm{~K} / \mathrm{min}$, we explicitly verify that the initial temperature of the cooling produces no significant change in the domain-vortex density if the cooling starts above said value of $T_{c}+20 \mathrm{~K}$. Only the cooling rate itself matters in that case. Additionally, we find that, at least up to $20 \mathrm{~K}$ below $T_{c}$, postannealing runs produce no essential change in the ferroelectric domain structure (see also Ref. [24]).

Taking all these precharacterization results into account, the subsequent cooling-rate-dependent experiments are performed by heating samples to between 40 and $210 \mathrm{~K}$ above $T_{c}$ and subjecting them to cooling rates ranging from $10^{-2}$ to $\approx 10^{5} \mathrm{~K} / \mathrm{min}$ down to between 50 and $460 \mathrm{~K}$ below $T_{c}$ (details in Appendix B). To exclude surface-related effects, samples are then thinned by about $100 \mu \mathrm{m}$ before polishing them and resolving the ferroelectric domain structure by $\mathrm{PFM}$ at room temperature. The resulting images are shown for $\mathrm{DyMnO}_{3}$ and $\mathrm{ErMnO}_{3}$ in Fig. 2; the behavior of $\mathrm{YMnO}_{3}$ is similar. Both compounds exhibit the characteristic domain pattern in which meeting points

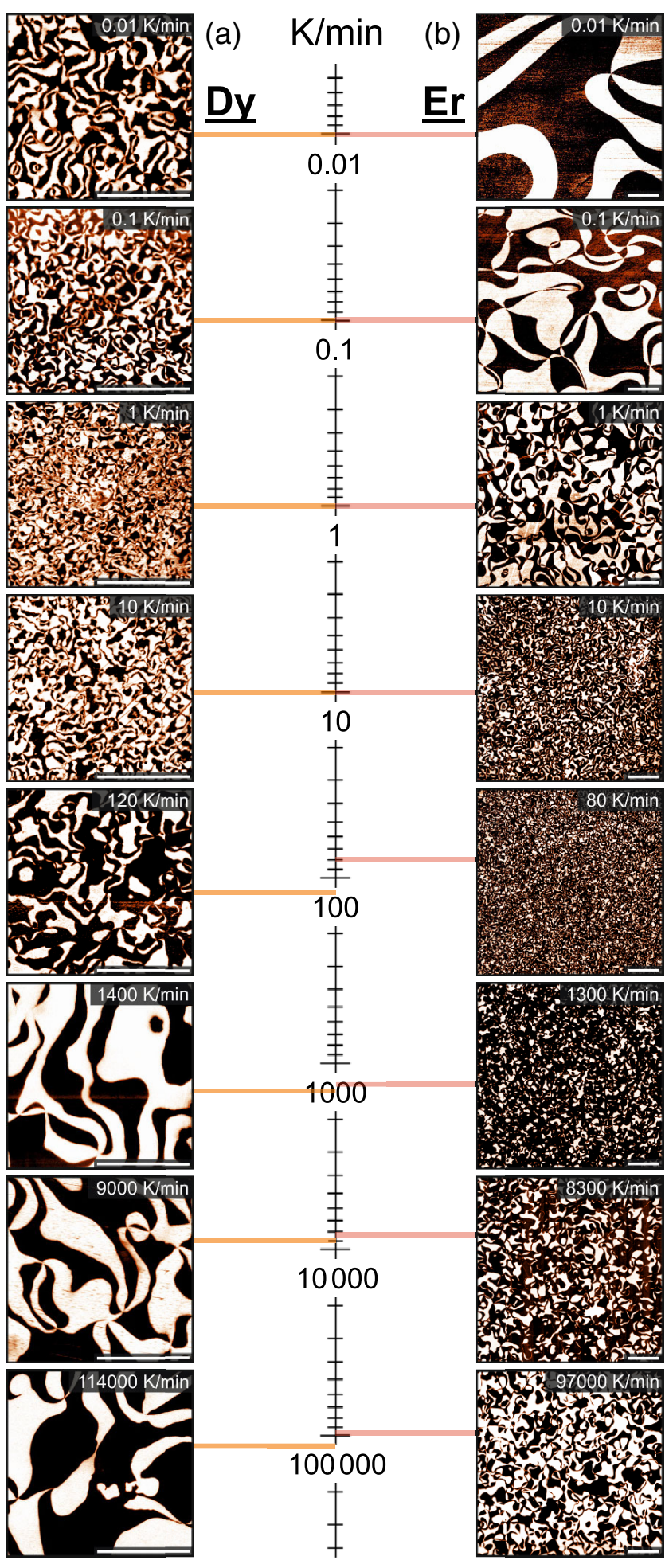

FIG. 2. Spatial maps of the vortexlike ferroelectric domain pattern in hexagonal manganites for a range of different cooling rates through $T_{c}$. Images were recorded by PFM under ambient conditions. Labels denote the respective cooling rates. Scale bar in all images is $5 \mu \mathrm{m}$. (a) $\mathrm{DyMnO}_{3}$. (b) $\mathrm{ErMnO}_{3}$. The KibbleZurek-like increase of vortex density with cooling rate, followed by a reversal and decrease of vortex density, is clear in both compounds. 


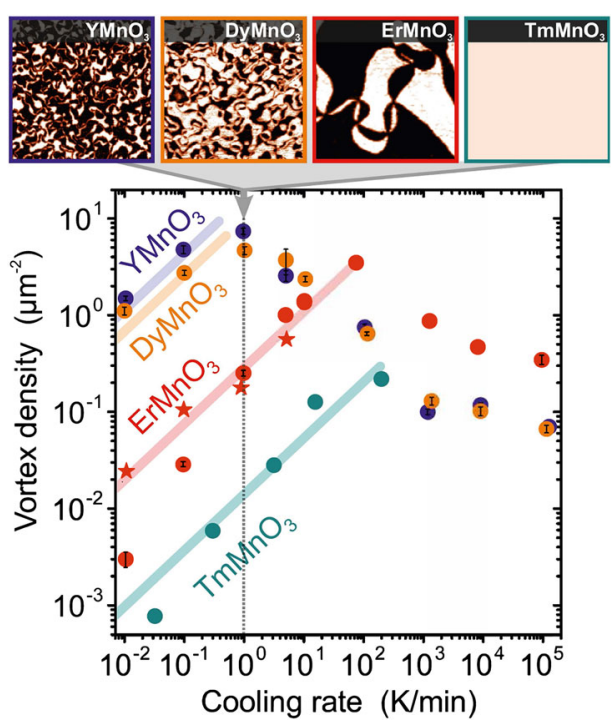

FIG. 3. Dependence of domain vortex density on cooling rate through $T_{c}$ across the $R \mathrm{MnO}_{3}$ series for $R=\mathrm{Y}$, Dy, Er, Tm. Two regimes, one in which the vortex density increases and one in which it decreases with cooling rate, are obvious. Lines show the fitted Kibble-Zurek behavior. Error bars represent the statistical error of the counted vortex number. Data for $\mathrm{TmMnO}_{3}$ were taken from Ref. [13]. Starlike red symbols indicate data points taken on flux-grown samples in Ref. [12]. Insets show PFM images of the domain structure after quenching at $1 \mathrm{~K} / \mathrm{min}$ for $5 \times 5 \mu \mathrm{m}^{2}$ sections. The $\mathrm{TmMnO}_{3}$ image is sketched as its domain size would exceed the shown section.

of the six trimerization-polarization domains identify the location of the topological vortex defects. $\mathrm{DyMnO}_{3}$ shows an increase of vortex density $n$ up to a cooling rate of $1 \mathrm{~K} / \mathrm{min}$, followed by a striking decrease of $n$ by 2 orders of magnitude upon further increase of the cooling rate. $\mathrm{ErMnO}_{3}$ displays qualitatively the same behavior, but the decrease of $n$ sets in at higher cooling rates than in $\mathrm{DyMnO}_{3}$. Note that previous experiments $[12,13]$ were performed at slower cooling rates, and so did not discover the turnaround in the slope of $n$.

The vortex density as a function of cooling rate for all four $R \mathrm{MnO}_{3}$ compounds is displayed in Fig. 3, clearly revealing that both the Kibble-Zurek and the anti-KibbleZurek behavior are generic features of the vortex formation in the hexagonal manganites. We also find an intriguing dependence on the crystal chemistry, with larger $R^{3+}$ radius correlating with higher $n$ at a given cooling rate within the Kibble-Zurek region, as well as with a decrease of the cooling rate at which the turnaround occurs. In addition, we observe deviations from Kibble-Zurek scaling in the ultraslow-cooling regime to which we return in Sec. III. Note that variations of the temperature at which we begin and end the controlled cooling above and below $T_{c}$, respectively, according to Appendix $\mathrm{B}$, have no influence on the data. This becomes particularly clear by the similarity of the data for $\mathrm{YMnO}_{3}$ and $\mathrm{DyMnO}_{3}$ for which the controlled cooling is maintained down to 500 and $50 \mathrm{~K}$, respectively, below $T_{c}$. This confirms that the temperature intervals of the quenchings are wide enough to observe Kibble-Zurek physics.

\section{Global Kibble-Zurek mechanism}

We now discuss the origin of the turnaround between Kibble-Zurek and anti-Kibble-Zurek behavior. According to the Kibble-Zurek mechanism, the vortices are expected to emerge from critical fluctuations with a density that is essentially determined by the rate of cooling through the phase transition and the critical slowing-down of the system [28]. When the relaxation of the order parameter becomes slower than the changes introduced by the quenching, the thermal fluctuations freeze out and give rise to a nonequilibrated order-parameter distribution.

We first revise this picture by making a distinction between two fluctuation regimes, namely, the Gaussian (about mean-field) and the Ginzburg (or scaling) regimes [29-31]. Roughly speaking, fluctuations are assumed to be noninteracting fluctuations in the Gaussian approximation while their interaction becomes crucial and controls the critical properties in the Ginzburg regime. These fluctuations determine the vortex density $n$ expected according to the Kibble-Zurek hypothesis as

$$
n \sim \frac{1}{\xi^{2}\left(t_{*}\right)}=\frac{1}{\xi_{0}^{2}}\left(\frac{\tau_{0}}{\tau_{q}}\right)^{2 \nu /(1+z \nu)} .
$$

Here, $\xi\left(t_{*}\right)$ is the coherence length at the freeze-out time $t_{*}$, which in turn is the time at which the quenching process becomes faster than the relaxation of the order parameter $\tau$. For a linear quench with $T(t)=\left(1-t / \tau_{q}\right) T_{c}$, where $\tau_{q}$ is the characteristic time set by the cooling rate $r=T_{c} \tau_{q}^{-1}$, the freeze-out time is given by $t_{*} \sim\left(\tau_{0} \tau_{q}^{z \nu}\right)^{1 /(1+z \nu)}$ [2,20]. Here, $z$ and $\nu$ are the dynamical critical exponent and the critical exponent for the correlation length according to $\tau(\varepsilon)=$ $\tau_{0} /|\varepsilon|^{z \nu}$ and $\xi(\varepsilon)=\xi_{0} /|\varepsilon|^{\nu}$, where $\varepsilon=\left(T-T_{c}\right) / T_{c}$ is the (time-dependent) reduced temperature [32-34].

We see in Eq. (2) that the precise form of the KibbleZurek scaling is fundamentally related to the nature of the critical fluctuations at the freeze-out. In the mean-field regime the critical exponents are $\nu=1 / 2$ and $z=2$, which leads to a mean-field Kibble-Zurek exponent $2 \nu /(1+z \nu)=1 / 2$. In addition, the microscopic correlation length is $\xi_{0}=\sqrt{s / a_{0}}$ in terms of the Landau freeenergy parameters in Eq. (1) with the expansion $a=-a_{0} T_{c} \varepsilon$. On the other hand, the critical exponents for Eq. (1) in the Ginzburg regime take the values $\nu=$ 0.672 and $z \simeq 2$ of the 3D $X Y$ model $[13,24,35,36]$. As a result, the Kibble-Zurek exponent becomes $2 \nu /(1+z \nu)=$ 0.58 . The onset of interaction between fluctuations characterizing the transition to the Ginzburg regime modifies 
not only the critical exponents but also "microscopic" parameters such as $\xi_{0}$, which becomes

$$
\tilde{\xi}_{0}=\left(\frac{5 b k_{B} T}{\pi^{2} \bar{s}^{2}} \xi_{0}\right)^{2 \nu / 5} \xi_{0}
$$

where $\bar{s}$ is the relevant parameter determining the gradient stiffness (see Appendix D).

The crossover between the mean-field and Ginzburg regimes is defined by the Ginzburg-Levanyuk criterion as the point at which the order-parameter fluctuations in a correlation volume of size $\xi^{3}$ reach the magnitude of the order parameter itself [29-31]. This crossover can be more accurately estimated from direct observables such as the specific heat. Thus, with Eq. (1) one obtains the so-called Ginzburg-Levanyuk number $\mathrm{Gi} \equiv \varepsilon_{\text {crossover }}=$ $\left(b k_{B} T_{c}\right)^{2} /\left[(4 \pi)^{2} a_{0} \bar{s}^{3}\right][30]$.

This has to be compared with the reduced freeze-out temperature $\varepsilon\left(t_{*}\right)=\left(\tau_{0} / \tau_{q}\right)^{1 /(1+z \nu)}$ to determine the formation of vortices in the Kibble-Zurek picture. At slow cooling rates, where $\varepsilon\left(t_{*}\right)<\mathrm{Gi}$, the Kibble-Zurek mechanism probes the fluctuation-dominated Ginzburg region. However, as the cooling rate increases, the freeze-out eventually occurs so far from $T_{c}$ that $\varepsilon\left(t_{*}\right)>\mathrm{Gi}$ and, hence, the Kibble-Zurek physics emerges from Gaussian fluctuations in the mean-field regime. The critical exponents and the microscopic parameters then change accordingly.

The expected crossover for a system described by Eq. (1) is illustrated in Fig. 4. Since the Kibble-Zurek exponent $2 \nu /(1+\nu z)$ is larger in the Ginzburg regime than in the mean-field regime, an offset between the related lines in the double-logarithmic plot is expected. The density of defects can then be expected to show a dropdown if the transition

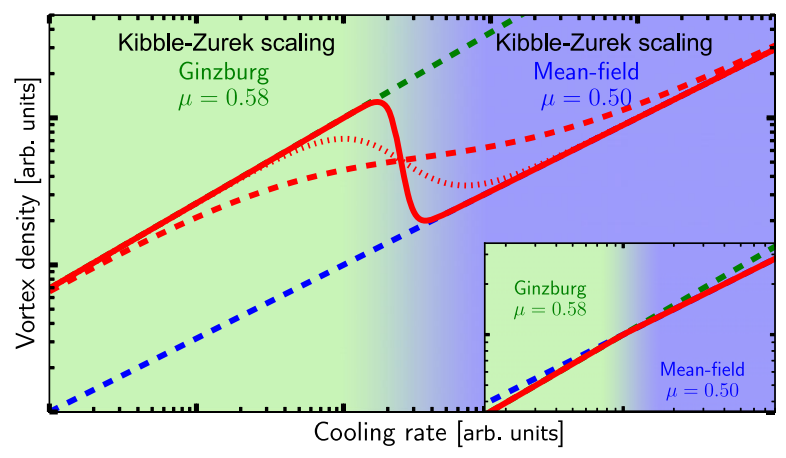

FIG. 4. Evolution of the Kibble-Zurek scaling $[\mu=2 \nu /(1+z \nu)]$. As the fluctuation regime probed in the experiment changes from Ginzburg (green dashed lines) to mean-field (blue dashed lines) behavior, the density of defects can display either a dropdown behavior (solid red line), a steplike transition (broken red lines), or a smooth transition (solid red line in the inset). The scenario depends on the microscopic parameters of the system, foremost the renormalization of the relaxation time $\tau_{0}$ when entering the Ginzburg regime (see text). from the Ginzburg to the mean field is sufficiently abrupt (see Fig. 4, red solid line, in contrast to the softer transitions shown as red broken lines). The observed scenario eventually depends on the particular system under consideration.

The values for Gi obtained from DFT calculations (see Table I) justify the slow-cooling scenario of the Ginzburg regime with its value of 0.58 for the Kibble-Zurek exponent. Note that the Gi values in Table I are unusually large (see, e.g., Ref. [37]) and could imply unphysically high crossover temperatures. Smaller, possibly more realistic values of $\mathrm{Gi}$ are obtained if we assume that the relevant stiffness controlling the fluctuations is $s_{z}$ instead of the averaged value $s$, which may be justified in view of the anisotropy of our system. This replacement would also reduce the resulting crossover temperature. Thus, the expected crossover in the Kibble-Zurek scaling is fully compatible with the dropdown observed in our experiments.

Two additional effects determine the relative position of the Ginzburg and mean-field asymptotic lines in Fig. 4. On the one hand, we see in Table I that the hexagonal manganites have a consistently larger correlation length $\tilde{\xi}_{0}$ in the Ginzburg than in the mean-field regime, $\xi_{0}$. This should tend to reduce the offset shown in Fig. 4 and could even lead to a crossing of the Ginzburg and mean-field graphs (Fig. 4, inset), along with a smooth transfer between the regimes as indicated by the line. On the other hand, a central factor discriminating between the two scenarios is the renormalization of the relaxation time $\tau_{0}$. Equation (3) shows that the correlation length increases in the Ginzburg regime, and with this we expect the relaxation time $\tau_{0}$ to go up. Hence, the dropdown scenario is the more likely one, providing an explanation for our experimental results in Figs. 2 and 3. We expect that experiments at higher quench rates, which we have not yet been able to access, should show a second turnaround, as vortex densities begin once again to increase with quench rate following a mean-field scaling.

Note that, in the seemingly unrelated case of a quantum phase transition driven by a noisy control parameter, the possibility of a dropdown has also been pointed out [38]. The analogy between this system and our system is that, in both cases, the departure from the Kibble-Zurek scaling is due to the running of the critical exponents-which, in Ref. [38], change from their nominal to their noise-limited value.

\section{DISCUSSION}

We first discuss the deviations observed in our $\mathrm{ErMnO}_{3}$ samples in the ultraslow-cooling regime. As shown in Fig. 3, here the vortex density increases but without reaching expected value. Such behavior was already noticed for other systems [4,14,39] and attributed to vortex-antivortex annihilation. This explanation is consistent with our finding that the falloff is largest at very slow 
cooling, when the time spent close to $T_{c}$ is large. It is also consistent with its absence in the flux-grown samples of Ref. [12]; our samples are grown using the floating-zone method, which we expect to have distinctly different stoichiometric modifications. We indeed observe that the mobility of vortices below $T_{c}$ can vary substantially among samples depending on the annealing gas atmosphere.

We now analyze the pronounced trends with the chemistry across the $R \mathrm{MnO}_{3}$ series that are clear in Fig. 3. In particular, the vortex density for a given cooling rate in the slow-cooling regime increases by 3 orders of magnitude from $\mathrm{Tm}$ to Dy, i.e., with decreasing $R^{3+}$ radius. In the Kibble-Zurek picture this is directly related to the microscopic parameters $\xi_{0}$ and $\tau_{0}$. Our first-principles calculations (see Table I) indicate that the mean-field value for $\xi_{0}$ is essentially the same in the four systems, as is the renormalized value $\tilde{\xi}_{0}\left(\simeq 1.3 \xi_{0}\right)$ in the fluctuation regime. Therefore, to obtain a factor $\sim 10^{3}$ in the vortex density in either the conventional mean-field or fluctuation-dominated Kibble-Zurek picture, $\tau_{0}$ would need to change by an unphysical factor of $\sim 10^{6}$. Strictly speaking, the renormalization of $\xi_{0}$ should be computed at the freeze-out temperature $T_{*}=\left[1+\left(\tau_{0} / \tau_{q}\right)^{1 /(1+z \nu)}\right] T_{c}$. However, this does not help us to reconcile the differences, since to increase the vortex density by the observed factor $10^{3}$, the freeze-out temperature would have to reach $T_{*} \sim 100 T_{c} \gtrsim 10^{5} \mathrm{~K}$. This is again unphysical. We thus see that the vortex formation in the $R \mathrm{MnO}_{3}$ systems displays quantitative features challenging the interpretation in terms of the original Kibble-Zurek mechanism. Specifically, the trend with chemistry does not fit with the standard scenario, even with the role of critical fluctuations beyond the mean-field description fully taken into account.

Another trend in Fig. 3 is the several-orders-of-magnitude shift of the cooling rate at which the departure from the initial Kibble-Zurek scaling occurs and which is not predicted by the calculated values for $\mathrm{Gi}$ in Table I. Specifically, the Gi number turns out to be essentially constant across the $R \mathrm{MnO}_{3}$ series even if it has the usual strong dependence on the relevant gradient parameter [37]. At the same time, it is striking that the huge spread of the vortex density in the Kibble-Zurek range is substantially reduced in the anti-Kibble-Zurek regime (from 1000 to 4), and, in fact, almost restores the approximate $R$ independence expected from Table I.

At least in part, extrinsic factors may be responsible for these unexpected trends with chemistry. Temperaturedependent processes related to chemical or mechanical impurities could affect the vortex formation differently across the $R \mathrm{MnO}_{3}$ series, simply because of the substantial increase of $T_{c}$ from Dy to Tm. In addition, the chemical and mechanical quality of the samples may change with $R$. $\mathrm{DyMnO}_{3}$, in particular, is much harder to grow hexagonally than, e.g., $\mathrm{TmMnO}_{3}$ because of the closer proximity of the competing perovskite phase. In fact, we find that batches of the same material grown under slightly different conditions can show, at a specific cooling rate, a vortex-density spread up to factor 4 with overall trends with cooling rate like in Fig. 3 (see Appendix B).

These factors can modify, in particular, the thermal conductivity of the samples. This conductivity is another ingredient limiting the overall thermalization of the system, and is such that the instantaneous temperature can additionally be position dependent across the sample. If this happens, the local transitions will not be simultaneous during the experiments. Instead, there will be a phase-transition front propagating at the speed $v_{f}=$ $\left.[(d x) /(d t)]\right|_{T(x, t)=T_{c}}$, which needs to be compared with the characteristic speed $v_{c}=\xi(\varepsilon) / \tau(\varepsilon)=\left(\xi_{0} / \tau_{0}\right)|\varepsilon|^{z}$ of the order-parameter relaxation [40-43]. Thus, if the front propagates much slower than the order-parameter perturbations, the formation defects can be heavily suppressed in a composition-dependent fashion.

Finally, we briefly discuss other possible origins of the anti-Kibble-Zurek behavior. The thermal conductivity could be one of them since, according to the above, a maximum in the vortex density can be expected at $v_{f}=v_{c}$, followed by a quick decrease as the cooling rate (and, hence, $v_{c}$ ) increases. On the other hand, the reversal from Kibble-Zurek to anti-Kibble-Zurek scaling has also been demonstrated in a recent Bose-Einstein-condensate transition experiment [15]. In that case, it has been ascribed to vortex-vortex repulsive interactions. Such interactions could also play a role in our $R \mathrm{MnO}_{3}$ systems, which certainly display a rather dense vortex pattern in the crossover region (see Fig. 2). In addition, there are additional time scales that can become relevant in the problem. The most obvious one is the time scale needed to obtain a fully developed vortex from the initial seed. Indeed, by increasing the cooling rate, this process can be expected to be gradually suppressed-simply because the phonons will not have time to propagate the order-parameter perturbations [44] — which will eventually lead to a decrease in the vortex density.

Thus, the overall trend in Fig. 3 may still be largely determined by intrinsic properties, and, hence, directly related to the corresponding universality class. In this regard, the discrete nature of the symmetry breaking can play a crucial role, especially if, as has been repeatedly underlined (see, e.g., Refs. [45-47]), the defect formation is decided after crossing the transition temperature. In fact, a generic feature of $Z_{6}$ models like Eq. (1) is the emergence of a second correlation length below $T_{c}$ associated with the phase of the order parameter supplementing that of its amplitude [24]. This, however, is missed in the usual Kibble-Zurek mechanism formulated for U(1) models. This second correlation length diverges faster than the standard correlation length [24]. Thus, if the critical slowing-down of the phase itself becomes a dominant process, the vortex density might become affected in a substantial way. 


\section{CONCLUSION}

In summary, we show theoretically that the Kibble-Zurek picture inherently includes a transfer from the asymptotic scaling, where the system is well inside the Ginzburg region, to mean-field scaling, where fluctuations represent small perturbations. This transfer is general since all phase transitions, from the interatomic to the cosmological scale, are formally expected to undergo such a crossover. We match our predictions with the experimental behavior in the series of hexagonal manganites and show that this crossover can be behind the striking reversal of the topological defect density as a function of the cooling rate (anti-KibbleZurek scaling). In addition, using density functional theory, we quantify the expected Kibble-Zurek behavior across the $R \mathrm{MnO}_{3}$ series. This quantification reveals the presence of sizable chemical trends that call for a vital upgrade of the original Kibble-Zurek considerations for the systems in this class.

\section{ACKNOWLEDGMENTS}

We thank A. Varlamov for carefully checking Eq. (3), Thomas Weber for support concerning powder X-ray diffraction, which was performed at the X-Ray Service Platform of the Department of Materials of the ETH Zurich, and Thomas Lottermoser and Andrea Scaramucci for fruitful discussions. This work was supported by the ERC Advanced Grant No. 291151 CCICO. Computational resources were provided by ETHZ and by a grant from the Swiss National Supercomputing Centre (CSCS) under Project No. p504. E. K. H. S. is grateful to EPSRC for support (Grant No. EP/P024904/1). S. M. G. is supported by the Laboratory Directed Research and Development Program at the Lawrence Berkeley National Laboratory under Contract No. DE-AC02-05CH11231, and by the Molecular Foundry through the DOE, Office of Basic Energy Sciences under the same contract number. A. C. is grateful to the French Government "Investments for the Future" Program, University of Bordeaux Initiative of Excellence (IDEX Bordeaux) for support.

Q. N. M. and M. L. contributed equally to this work.

\section{APPENDIX A: GROWTH AND CHARACTERIZATION OF $\mathrm{DyMnO}_{3-\delta}$ SAMPLES $(\delta \approx 0.05)$}

Hexagonal crystalline $\mathrm{DyMnO}_{3-\delta}$ is prepared by the optical floating-zone melting technique using a Cyberstar mirror furnace as follows.

The starting materials are $\mathrm{Dy}_{2} \mathrm{O}_{3}$ powder (abcr $\mathrm{GmbH}$, 99.99\% rare-earth oxide basis) and $\mathrm{Mn}_{2} \mathrm{O}_{3}$ powder (MaTeck, 99.9\% metals basis). Any moisture in the starting materials is removed by heating them at elevated temperatures under air in a laboratory chamber furnace $\left(\mathrm{Mn}_{2} \mathrm{O}_{3}\right.$ powder up to $400^{\circ} \mathrm{C}, \mathrm{Dy}_{2} \mathrm{O}_{3}$ up to $1100^{\circ} \mathrm{C}$ ) for a dwell time of at least $1 \mathrm{~h}$. In addition, the chemical composition of the $\mathrm{Mn}_{2} \mathrm{O}_{3}$ powder is confirmed using thermogravimetric analysis.

A 1:1 molar ratio of mingled $\mathrm{Dy}_{2} \mathrm{O}_{3}$ and $\mathrm{Mn}_{2} \mathrm{O}_{3}$ powders is pre-reacted in an alumina crucible overnight up to $1250^{\circ} \mathrm{C}$ (dwell time $4 \mathrm{~h}$ ) under air. The sintered powder is then ground and pressed into rods which are sintered for $4 \mathrm{~h}$ at $1310^{\circ} \mathrm{C}$ under air. Next, the polycrystalline sintered rods are processed by floating-zone melting in a Cyberstar mirror furnace under flowing argon (99.999\%, flow rate 24 liter/h) and with a zone speed or growth rate of $8 \mathrm{~mm} / \mathrm{h}$.

The oxygen content of argon at the gas outlet of the Cyberstar mirror furnace is monitored using an oxygen analyzer. During the creation of the molten zone an oxygen content of about $400 \mathrm{ppm}$ is detected for a short time. This decreases steadily during the first hour of growth so that after $1 \mathrm{~h}$ and during the subsequent $4 \mathrm{~h}$ the detected oxygen content is always in a range of about $50-40 \mathrm{ppm}$. This enhanced oxygen content of the argon at the outlet of the mirror furnace indicates that the original chemical composition $\mathrm{DyMnO}_{3}$ has released oxygen resulting in a meltgrown sample with composition $\mathrm{DyMnO}_{3-\delta}$ with $\delta>0$. This is confirmed by thermogravimetric analysis, which indicates $\delta \approx 0.05$.

Powder x-ray diffraction of melt-grown and pulverized $\mathrm{DyMnO}_{3-\delta}$ is performed with a Panalytical X'Pert PRO MPD diffractometer equipped with a Ge monochromator ( $\mathrm{Cu} K \alpha_{1}$ radiation). The measured powder x-ray pattern of the melt-grown $\mathrm{DyMnO}_{3-\delta}$ displays 31 peaks within the angle range $3^{\circ} \leq 2 \Theta \leq 73^{\circ}$. All observed peaks fit to a hexagonal $\mathrm{YMnO}_{3}$-type structure. Lattice parameter refinement gives $a=b=6.18 \AA, c=11.47 \AA$, and $V=379.3 \AA^{3}$ ( $P$-type Bravais lattice). The modulus of the difference between the observed and calculated peak position is $\leq 0.035^{\circ}$ for all observed peaks.

The applied procedures and used devices such as the custom-made agate mortar and pestle, special and custommade pressing dies, preparation of the feed and seed rod, mirror furnace, special sample holders for the feed and seed rod, oxygen analyzer, videos from the floating-zone melting process, and pictures of melt-grown crystalline DyMnO${ }_{3}$ are presented in detail in Ref. [48].

\section{APPENDIX B: SAMPLE PREPARATION}

Samples are grown at ETH $\left(\mathrm{DyMnO}_{3}\right)$, PSI $\left(\mathrm{YMnO}_{3}\right)$, and Lawrence Berkeley National Laboratory $\left(\mathrm{ErMnO}_{3}\right)$ by the FZ technique as described in Appendix A and elsewhere [49-51]. Rods are oriented by Laue diffraction and cut into $z$-oriented platelets of a few $\mathrm{mm}$ lateral size and a thickness of about $0.7 \mathrm{~mm}$.

For minimizing sample-dependent drifts in measurements of the cooling-rate-dependent ferroelectric domain vortex density, all samples of a batch are preannealed under identical conditions by heating above $T_{c}$ for several hours 
$\left(\mathrm{YMnO}_{3}, 1400 \mathrm{~K} ; \mathrm{DyMnO}_{3}, 1270 \mathrm{~K} ; \mathrm{ErMnO}_{3}, 1470 \mathrm{~K}\right)$ and cooling through $T_{c}$ at a rate of $5 \mathrm{~K} / \mathrm{min}$. To reveal the intrinsic 3D bulk domain structure and suppress surfacedependent effects, samples are then thinned by at least $100 \mu \mathrm{m}$ by lapping with $\mathrm{Al}_{2} \mathrm{O}_{3}$ powder. This is followed by etch polishing with a silica slurry, revealing shiny surfaces with a root-mean-square roughness below $1 \mathrm{~nm}$ before determining the vortex density by PFM.

The PFM characterization of the preannealed specimens reveals that the vortex density changes systematically with the location in the original FZ-grown rod from which the samples are extracted. A variation of up to a factor 2 was observed for a distance of $10 \mathrm{~mm}$ along the growth direction of the rod. As described in the main text, we therefore preselect only those samples that reveal, within statistical error, the same density of vortices under identical annealing conditions.

The quench experiments are performed like the preannealing experiments but with a dwell time above $T_{c}$ of few tens of minutes and varying cooling rate through $T_{c}$. In order to avoid accumulation of chemical drift occurring during the quench cycles, each data point is gained from a different specimen of the preselected set. The range across which the specified cooling rate is maintained is as follows: $\mathrm{DyMnO}_{3}\left(T_{c}=1223 \mathrm{~K}\right), 1270-1170 \mathrm{~K}$ for cooling rates $<100 \mathrm{~K} / \mathrm{min}$ and $1420-1170 \mathrm{~K}$ for cooling rates $>100 \mathrm{~K} / \mathrm{min} ; \mathrm{YMnO}_{3}\left(T_{c}=1259 \mathrm{~K}\right), 1460-970 \mathrm{~K}$; $\mathrm{ErMnO}_{3}\left(T_{c}=1429 \mathrm{~K}\right), 1470-970 \mathrm{~K}$.

\section{APPENDIX C: FIRST-PRINCIPLES CALCULATIONS}

For our density functional calculations we use the projector-augmented wave method as implemented in the ABINIT code [52-55]. We use a plane-wave cutoff of $30 \mathrm{Ry}$ and a $6 \times 6 \times 2 k$-point grid. To take into account correlation effects on the Mn atoms, we use the LDA $+\mathrm{U}$ method within the fully localized limit method as introduced in Refs. [56,57]. We choose a value of $U$ of $8 \mathrm{eV}$ and $J=0.88 \mathrm{eV}$. For all our calculations we adopt an $A$-type magnetic ordering of the $\mathrm{Mn}$ ions and freeze the rare-earth $f$ electrons in the pseudopotential cores. To extract the parameters in the Landau free energy, Eq. (1), we first fully relax the $P 6_{3} / m m c$ structure to an accuracy of $10^{-6} \mathrm{Ry} / \mathrm{bohr}$. We find unit-cell volumes of $364.73,364.42,367.50$, and $353.00 \AA^{3}$ for $\mathrm{YMnO}_{3}, \mathrm{DyMnO}_{3}, \mathrm{ErMnO}_{3}$, and $\mathrm{TmMnO}_{3}$, respectively. We then calculate the force constants using the finite displacement method and extract the eigenvectors of the force constant matrix. We then gradually freeze in the eigenvector of the unstable $K_{3}$ mode and fit a sixth-order polynomial to extract the $a_{0}$ and $b$ terms. To calculate the gradient term $s$ we exploit the fact that in $q$ space $s(\nabla Q)^{2}$ reduces to $s q^{2}\left|Q_{\mathbf{q}}\right|^{2}$ and $s$ can then be obtained by fitting a parabola to the corresponding branch of the force constant dispersion, as shown in Ref. [27]. We note that the values of the parameters are dependent on the choice of exchangecorrelation functional because $a$ scales quadratically and $b$ to the fourth power with the lattice constant. The trends across the series, however, are robust to the computational details.

\section{APPENDIX D: DERIVATION OF EQ. (3)}

Here, we derive Eq. (3) for the renormalized correlation length in the Ginzburg region.

First, we note that due to the actual anisotropy of the gradient term in Eq. (1), the integrals in Fourier space become

$$
\begin{aligned}
\int d^{3} k f\left(s k^{2}\right) & =\frac{1}{s^{3 / 2}} \int d^{3} q f\left(q^{2}\right) \rightarrow \\
\int d^{3} k f\left(s_{x} k_{x}^{2}, s_{x} k_{y}^{2}, s_{z} k_{z}^{2}\right) & =\frac{1}{\left(s_{x}^{2} s_{z}\right)^{1 / 2}} \int d^{3} q f\left(q_{x}^{2}, q_{y}^{2}, q_{z}^{2}\right) .
\end{aligned}
$$

This defines $\bar{s}=\left(s_{x}^{2} s_{z}\right)^{1 / 3}$ as the appropriate mean for the gradient coefficient, which we use in the following.

To derive Eq. (3) we follow Larkin and Varlamov [30]. Thus, we obtain the renormalization-group equations,

$$
\begin{gathered}
\frac{\partial a(T, \Lambda)}{\partial \Lambda}=-k_{B} T \mu_{D} \frac{b(\Lambda) \Lambda^{D-1}}{\frac{a(\Lambda)}{2}+\frac{s}{2} \Lambda^{2}}, \\
\frac{\partial b(T, \Lambda)}{\partial \Lambda}=\frac{5 k_{B} T \mu_{D}}{4} \frac{b^{2}(\Lambda) \Lambda^{D-1}}{\left(\frac{a(\Lambda)}{2}+\frac{s}{2} \Lambda^{2}\right)^{2}},
\end{gathered}
$$

for the parameters in the Landau free energy Eq. (1) (here and hereafter, $s$ has to be understood as $\bar{s}$ ). Here, $\mu_{D}=D /\left[2^{D} \pi^{D / 2} \Gamma(1+D / 2)\right]$, where $D$ is the dimensionality of the problem $(D=3$ in our case). Strictly speaking, these equations are valid close enough to $T_{c}$ in the Ginzburg region for $D \rightarrow 4$. Nevertheless, the critical behavior of our 3D system can be studied from these equations by means of the so-called $\epsilon$ expansion [30].

The generalized correlation length is defined by the equation

$$
\xi^{-2}(T)=\frac{\alpha\left(T_{c}, \xi^{-1}(T)\right)}{s} T_{c} \varepsilon,
$$

where $\alpha$ is determined from the expansion $a(T, \Lambda)=$ $a\left(T_{c}, \Lambda\right)+\alpha\left(T_{c}, \Lambda\right) T_{c} \varepsilon$. For this latter quantity, the selfconsistent solution of the renormalization-group equations gives [30]

$\alpha\left(T_{c}, \Lambda\right)=\alpha_{0}\left[1+\frac{10 k_{B} T \mu_{D}}{s^{2}(4-D)}\left(\Lambda^{D-4}-\xi_{0}^{4-D}\right)\right]^{-2 / 5}$.

As $T \rightarrow T_{c}$ the right-hand side of this expression is dominated by the term containing $\Lambda^{D-4}$. Accordingly, 
$\alpha\left(T_{c}, \xi^{-1}\right) \approx \alpha_{0}\left\{10 k_{B} T \mu_{D} /\left[s^{2}(4-D)\right]\right\}^{-2 / 5} \xi^{2(D-4) / 5}$ and, therefore,

$$
\xi^{-2} \approx \frac{\alpha_{0} T_{c}}{s}\left(\frac{10 k_{B} T \mu_{D}}{s^{2}(4-D)}\right)^{-2 / 5} \xi^{2(D-4) / 5} \varepsilon .
$$

By means of simple manipulations, this equation can be transformed into

$$
\xi=\xi_{0}\left(\frac{10 k_{B} T \mu_{D} b \xi_{0}^{(4-D)}}{s^{2}(4-D)}\right)^{1 /(1+D)} \varepsilon^{-\nu}
$$

where $\nu=1 /\{2[1-(4-D) / 5]\}$. Thus, applying this result to the $3 \mathrm{D}$ case, we obtain

$$
\xi=\xi_{0}\left(\frac{5 k_{B} T b}{\pi^{2} s^{2}} \xi_{0}\right)^{2 \nu / 5} \varepsilon^{-\nu},
$$

with $\nu=3 / 5$. Equation (3) directly follows from this expression.

[1] T. W. B. Kibble, Topology of Cosmic Domains and Strings, J. Phys. A 9, 1387 (1976).

[2] W.H. Zurek, Cosmological Experiments in Superfluid Helium?, Nature (London) 317, 505 (1985).

[3] I. Chuang, B. Yurke, R. Durrer, and N. Turok, Cosmology in the Laboratory: Defect Dynamics in Liquid Crystals, Science 251, 1336 (1991).

[4] S. Ducci, P. L. Ramazza, W. González-Viñas, and F. T. Arecchi, Order Parameter Fragmentation after a Symmetry-Breaking Transition, Phys. Rev. Lett. 83, 5210 (1999).

[5] P. C. Hendry, N. S. Lawson, R. A. M. Lee, Peter V.E. McClintock, and C. D. H. Williams, Generation of Defects in Superfluid ${ }^{4} \mathrm{He}$ as an Analogue of the Formation of Cosmic Strings, Nature (London) 368, 315 (1994).

[6] M. E. Dodd, P. C. Hendry, N. S. Lawson, Peter V.E. McClintock, and C.D.H. Williams, Nonappearance of Vortices in Fast Mechanical Expansions of Liquid ${ }^{4} \mathrm{He}$ through the Lambda Transition, Phys. Rev. Lett. 81, 3703 (1998).

[7] C. Bäuerle, Y. M. Bunkov, S. N. Fisher, H. Godfrin, and G. R. Pickett, Laboratory Simulation of Cosmic String Formation in the Early Universe Using Superfluid ${ }^{3} \mathrm{He}$, Nature (London) 382, 332 (1996).

[8] V. M. H. Ruutu, V. B. Eltsov, A. J. Gill, T. W. B. Kibble, M. Krusius, Yu G. Makhlin, B. Placais, G. E.. Volovik, and Wen $\mathrm{Xu}$, Vortex Formation in Neutron-Irradiated Superfluid ${ }^{3} \mathrm{He}$ as an Analogue of Cosmological Defect Formation, Nature (London) 382, 334 (1996).

[9] R. Carmi, E. Polturak, and G. Koren, Observation of Spontaneous Flux Generation in a Multi-JosephsonJunction Loop, Phys. Rev. Lett. 84, 4966 (2000).

[10] R. Monaco, Jesper Mygind, R. J. Rivers, and V. P. Koshelets, Spontaneous Fluxoid Formation in Superconducting Loops, Phys. Rev. B 80, 180501 (2009).
[11] S. M. Griffin, M. Lilienblum, K. T. Delaney, Y. Kumagai, M. Fiebig, and N. A. Spaldin, Scaling Behavior and Beyond Equilibrium in the Hexagonal Manganites, Phys. Rev. X 2, 041022 (2012).

[12] S. C. Chae, N. Lee, Y. Horibe, M. Tanimura, S. Mori, B. Gao, S. Carr, and S.-W. Cheong, Direct Observation of the Proliferation of Ferroelectric Loop Domains and VortexAntivortex Pairs, Phys. Rev. Lett. 108, 167603 (2012).

[13] S.-Z. Lin, X. Wang, Y. Kamiya, G.-W. Chern, F. Fan, D. Fan, B. Casas, Y. Liu, V. Kiryukhin, W. H. Zurek, C. D. Batista, and S.-W. Cheong, Topological Defects as Relics of Emergent Continuous Symmetry and Higgs Condensation of Disorder in Ferroelectrics, Nat. Phys. 10, 970 (2014).

[14] S.-W. Su, S.-C. Gou, A. Bradley, O. Fialko, and J. Brand, Kibble-Zurek Scaling and Its Breakdown for Spontaneous Generation of Josephson Vortices in Bose-Einstein Condensates, Phys. Rev. Lett. 110, 215302 (2013).

[15] S. Donadello, S. Serafini, T. Bienaimé, F. Dalfovo, G. Lamporesi, and G. Ferrari, Creation and Counting of Defects in a Temperature-Quenched Bose-Einstein Condensate, Phys. Rev. A 94, 023628 (2016).

[16] S. Ulm, J. Roßnagel, G. Jacob, C. Degünther, S. T. Dawkins, U. G. Poschinger, R. Nigmatullin, A. Retzker, M. B. Plenio, F. Schmidt-Kaler, and K. Singer, Observation of the KibbleZurek Scaling Law for Defect Formation in Ion Crystals, Nat. Commun. 4, 2290 (2013).

[17] K. Pyka, J. Keller, H. L. Partner, and R. Nigmatullin, Topological Defect Formation and Spontaneous Symmetry Breaking in Ion Coulomb Crystals, Nat. Commun. 4, 2291 (2013).

[18] X.-Y. Xu, Y.-J. Han, K. Sun, J.-S. Xu, J.-S. Tang, C.-F. Li, and G.-C. Guo, Quantum Simulation of Landau-Zener Model Dynamics Supporting the Kibble-Zurek Mechanism, Phys. Rev. Lett. 112, 035701 (2014).

[19] S. Deutschlander, P. Dillmann, G. Maret, and P. Keim, Kibble-Zurek Mechanism in Colloidal Monolayers, Proc. Natl. Acad. Sci. U.S.A. 112, 6925 (2015).

[20] A. Del Campo and W.H. Zurek, Universality of Phase Transition Dynamics: Topological Defects from Symmetry Breaking, Int. J. Mod. Phys. A 29, 1430018 (2014).

[21] N. B. Kopnin and E. V. Thuneberg, Time-Dependent Ginzburg-Landau Analysis of Inhomogeneous NormalSuperfluid Transitions, Phys. Rev. Lett. 83, 116 (1999).

[22] W.H. Zurek, Cosmological Experiments in Condensed Matter Systems, Phys. Rep. 276, 177 (1996).

[23] L. M. A. Bettencourt, N. D. Antunes, and W. H. Zurek, Ginzburg Regime and Its Effects on Topological Defect Formation, Phys. Rev. D 62, 065005 (2000).

[24] M. Lilienblum, T. Lottermoser, S. Manz, S. M Selbach, A. Cano, and M. Fiebig, Ferroelectricity in the Multiferroic Hexagonal Manganites, Nat. Phys. 11, 1070 (2015).

[25] T. Choi, Y. Horibe, H. T. Yi, Y. J. Choi, Weida Wu, and S.-W. Cheong, Insulating Interlocked Ferroelectric and Structural Antiphase Domain Walls in Multiferroic $\mathrm{YMnO}_{3}$, Nat. Mater. 9, 423 (2010).

[26] Q. Zhang, G. Tan, L. Gu, Y. Yao, C. Jin, Y. Wang, X. Duan, and R. Yu, Direct Observation of Multiferroic Vortex Domains in $\mathrm{YMnO}_{3}$, Sci. Rep. 3, 2741 (2013). 
[27] S. Artyukhin, K. T. Delaney, N. A. Spaldin, and M. Mostovoy, Landau Theory of Topological Defects in Multiferroic Hexagonal Manganites, Nat. Mater. 13, 42 (2014).

[28] L. D. Landau and I. M. Khalatnikov, On the Anomalous Absorption of Sound Near a Second Order Phase Transition Point, Dokl. Akad. Nauk SSSR 96, 469 (1954).

[29] L. D. Landau and E. M. Lifshitz, Statistical Physics (Elsevier Science, New York, 2013), Vol. 5.

[30] A. Larkin and A. Varlamov, Theory of Fluctuations in Superconductors (Oxford University Press, New York, 2005).

[31] B. A. Strukov and A. P. Levanyuk, Ferroelectric Phenomena in Crystals: Physical Foundations (Springer, New York, 1998).

[32] P. C. Hohenberg and A. P. Krekhov, An Introduction to the Ginzburg-Landau Theory of Phase Transitions and Nonequilibrium Patterns, Phys. Rep. 572, 1 (2015).

[33] E. K. H. Salje, Application of Landau Theory for the Analysis of Phase Transitions in Minerals, Phys. Rep. 215, 49 (1992).

[34] E. K. H. Salje, Kinetic Rate Laws as Derived from Order Parameter Theory I: Theoretical Concepts, Phys. Chem. Miner. 15, 336 (1988).

[35] D. R. Nelson, Coexistence-Curve Singularities in Isotropic Ferromagnets, Phys. Rev. B 13, 2222 (1976).

[36] M. Oshikawa, Ordered Phase and Scaling in $Z_{n}$ Models and the Three-State Antiferromagnetic Potts Model in Three Dimensions, Phys. Rev. B 61, 3430 (2000).

[37] V. L. Ginzburg, A. P. Levanyuk, and A. A. Sobyanin, Light Scattering Near Phase Transition Points in Solids, Phys. Rep. 57, 151 (1980).

[38] A. Dutta, A. Rahmani, and A. del Campo, Anti-KibbleZurek Behavior in Crossing the Quantum Critical Point of a Thermally Isolated System Driven by a Noisy Control Field, Phys. Rev. Lett. 117, 080402 (2016).

[39] D. J. Weir, R. Monaco, V. P. Koshelets, J. Mygind, and R. J. Rivers, Gaussianity Revisited: Exploring the Kibble-Zurek Mechanism with Superconducting Rings, J. Phys. Condens. Matter 25, 404207 (2013).

[40] A del Campo, T. W. B. Kibble, and W. H. Zurek, Causality and Non-Equilibrium Second-Order Phase Transitions in Inhomogeneous Systems, J. Phys. Condens. Matter 25, 404210 (2013).

[41] G. E. Volovik, Defect Formation in Inhomogeneous 2nd Order Phase Transition: Theory and Experiment, Physica (Amsterdam) 280B, 122 (2000).

[42] J. Dziarmaga, P. Laguna, and W. H. Zurek, Symmetry Breaking with a Slant: Topological Defects after an Inhomogeneous Quench, Phys. Rev. Lett. 82, 4749 (1999).
[43] T. W. B. Kibble and G. E. Volovik, On Phase Ordering Behind the Propagating Front of a Second-Order Transition, J. Exp. Theor. Phys. 65, 102 (1997).

[44] L. Zhang, E. K. H. Salje, X. Ding, and J. Sun, Strain Rate Dependence of Twinning Avalanches at High Speed Impact, Appl. Phys. Lett. 104, 162906 (2014).

[45] G. Karra and R. J. Rivers, Reexamination of Quenches in ${ }^{4} \mathrm{He}$ (and ${ }^{3} \mathrm{He}$ ), Phys. Rev. Lett. 81, 3707 (1998).

[46] R. J. Rivers, Slow ${ }^{4}$ He Quenches Produce Fuzzy, Transient Vortices, Phys. Rev. Lett. 84, 1248 (2000).

[47] N. D. Antunes, P. Gandra, and R. J. Rivers, Is Domain Formation Decided Before or After the Transition?, Phys. Rev. D 73, 125003 (2006).

[48] F. Lichtenberg, Presentation of a laboratory for the synthesis and study of special oxides and melt-grown crystalline materials, Research Collection of the ETH Zurich (2017), https://www.research-collection.ethz.ch/handle/20.500 $.11850 / 127690$.

[49] B. Roessli, S. N. Gvasaliya, E. Pomjakushina, and K. Conder, Spin Fluctuations in the Stacked-Triangular Antiferromagnet $\mathrm{YMnO}_{3}$, Pis'ma Zh. Eksp. Teor. Fiz. 81, 360 (2005) [JETP Lett. 81, 287 (2005)].

[50] V. Yu. Ivanov, A. A. Mukhin, A. S. Prokhorov, A. M. Balbashov, and L. D. Iskhakova, Magnetic Properties and Phase Transitions in Hexagonal $\mathrm{DyMnO}_{3}$ Single Crystals, Phys. Solid State 48, 1726 (2006).

[51] Z. Yan, D. Meier, J. Schaab, R. Ramesh, E. Samulon, and E. Bourret, J. Cryst. Growth 409, 75 (2015).

[52] X. Gonze, J. M. Beuken, R. Caracas, F. Detraux, M. Fuchs, G. M. Rignanese, L. Sindic, M. Verstraete, G. Zerah, F. Jollet, M. Torrent, A. Roy, M. Mikami, Ph. Ghosez, J. Y. Raty, and D.C. Allan, First-Principles Computation of Material Properties: The ABINIT Software Project, Comput. Mater. Sci. 25, 478 (2002).

[53] X. Gonze, A Brief Introduction to the ABINIT Software Package, Z. Kristallogr. 220, 558 (2005).

[54] M. Torrent, F. Jollet, F. Bottin, G. Zérah, and X. Gonze, Implementation of the Projector Augmented-Wave Method in the ABINIT Code: Application to the Study of Iron Under Pressure, Comput. Mater. Sci. 42, 337 (2008).

[55] B. Amadon, F. Jollet, and M. Torrent, $\gamma$ and $\beta$ Cerium: LDA + U Calculations of Ground-State Parameters, Phys. Rev. B 77, 155104 (2008).

[56] J. P. Perdew and Y. Wang, Accurate and Simple Analytic Representation of the Electron-Gas Correlation Energy, Phys. Rev. B 45, 13244 (1992).

[57] A. I. Liechtenstein, V. I. Anisimov, and J. Zaanen, DensityFunctional Theory and Strong Interactions: Orbital Ordering in Mott-Hubbard Insulators, Phys. Rev. B 52, R5467 (1995). 